\title{
Towards integration of general practitioner posts and accident and emergency departments: a case study of two integrated emergency posts in the Netherlands Rudolf B Kool $^{* \dagger}$, Daniel J Homberg ${ }^{\dagger}$ and Helen CM Kamphuis ${ }^{\dagger}$
}

Address: Quality and Safety Department, Prismant Institute for Research in Health Care, Papendorpseweg 65, 3528 BJ, Utrecht, the Netherlands Email: Rudolf B Kool* - tijn.kool@prismant.nl; Daniel J Homberg - jeroen.homberg@prismant.nl; Helen CM Kamphuis - helen.kamphuis@prismant.nl

* Corresponding author †Equal contributors

Published: 4 November 2008

BMC Health Services Research 2008, 8:225 doi: I0.I 186/1472-6963-8-225
Received: 29 May 2008

Accepted: 4 November 2008

This article is available from: http://www.biomedcentral.com/1472-6963/8/225

(c) $2008 \mathrm{Kool}$ et al; licensee BioMed Central Ltd.

This is an Open Access article distributed under the terms of the Creative Commons Attribution License (http://creativecommons.org/licenses/by/2.0), which permits unrestricted use, distribution, and reproduction in any medium, provided the original work is properly cited.

\begin{abstract}
Background: Accident and emergency (A\&E) departments and general practitioner (GP) posts are often used inappropriately, leading to overcrowding. In the Netherlands, increasingly more integrated emergency posts (IEPs) are being created, integrating the care provided by GP posts and A\&E departments, in order to improve the provision of the emergency care.
\end{abstract}

Methods: This explorative study compares the efficiency and patient and employee satisfaction in IEPs with those in two GP posts and two A\&E departments. To this end, information was retrieved from hospital and GP patient records for the first quarter of the year before and of the year after the creation of IEPs. Patients and employees were sent a questionnaire to measure their satisfaction. Lastly, groups of hospital doctors, GPs, GP assistants, and nurses were interviewed.

Results: After the creation of IEPs, there was a shift of more than fifteen percent from secondary care to primary care for emergency consultations and waiting/consultation times were shortened by more than ten percent. Compared with the control settings, patients were more satisfied about telephone contact with an IEP, but professionals working at the IEP were less satisfied with several aspects of their work.

Conclusion: IEPs could be a promising innovation to organize emergency care more efficiently; however, it might take time to convince professionals of the possible advantages. Studies involving more IEPs and longer follow-up times are needed to determine whether such integration should be stimulated.

\section{Background}

In many countries accident and emergency (A\&E) departments have a problem with inefficient patient flow because of overcrowding [1-8]. An important factor contributing to this overcrowding is self-referral: increasingly more patients go directly to A\&E departments for out-ofhours services without first contacting their general practi- tioner (GP). At the same time, most of the problems seen in $\mathrm{A} \& \mathrm{E}$ departments are in principal primary care problems and can be regarded as inappropriate use of $\mathrm{A} \& \mathrm{E}$ services. Various studies report that between $15 \%$ and $50 \%$ of patients make inappropriate use of A\&E services [9-18]. Most of these patients can be treated by a GP or nurse and at lower cost [19-26]. 
In the Netherlands, some GP posts were set up in the vicinity of a hospital or in the hospital itself, in an attempt to reduce self-referrals and inappropriate use of hospital services $[27,28]$. More recently, a GP post and a hospital in two regions (Purmerend and Haarlem) took a next step to optimize patient flow at local A\&E departments by integrating the care provided by A\&E departments and GP posts, forming so-called integrated emergency posts (IEPs). The purpose of these posts is to provide appropriate treatment within one organisation with complex, specialized care being provided by A\&E doctors and less complex care being provided by GPs or specially trained nurses, such as nurse practitioners or physician assistants. In this way, it is expected that IEPs will shift the provision of care for certain health problems back from secondary care to primary care while providing patients with appropriate care for their problems.

To date (August 2008), seven IEPs have been established in the Netherlands and in several other regions A\&E departments and GP posts are preparing to integrate their services. As similar innovations have not been described in the literature, we investigated whether IEPs meet expectations. To this end, we used data from the IEPs in Purmerend and Haarlem and two control locations to determine (i) whether an IEP changes patient flow by reducing the number of patients seen by $A \& E$ staff and by increasing the number of patients seen by the GP or nurse; (ii) whether waiting/consultation times are shorter, thereby speeding up the flow of patients through emergency services; (iii) whether patients treated at an IEP are more satisfied than patients treated at a GP post or A\&E department; and (iv) whether doctors, nurses, and other professionals are satisfied with the concept of an IEP.

\section{Methods}

\section{Sites}

This study compares two IEPs with two comparable control settings, namely, traditional GP posts and A\&E departments. In these control settings, patients with health problems occurring out of hours decide themselves whether to phone their GP or to visit the local A\&E department. Patients phoning a GP post undergo triage by a GP assistant according to a protocol set up by the Dutch Association of General Practitioners. This triage is supervised by a GP. Possible outcomes of this triage are: self-care advice, advice to visit the own GP the next day, advice to visit the $\mathrm{A} \& \mathrm{E}$ department at the hospital, advice to visit the GP post to be seen by a GP or to been seen at home by a GP. Patients who come directly to a GP post are seen by the GP after registration and triage by the GP assistant. In the $\mathrm{A} \& \mathrm{E}$ department, a resident sees referred as well as self-referred patients after triage by an A\&E nurse. Most Dutch A\&E departments use the Manchester Triage System $[29,30]$.

When the IEPs were set up, patients were informed about the changes to emergency care provision by means of leaflets, posters, and media coverage. At the IEP, patients check in at the joint reception of the GP post and the A\&E department or call one telephone number. Triage is performed by a trained GP assistant (Purmerend) or nurse (Haarlem), using a standard protocol. She or he allocates the patient either to the A\&E doctor, the GP or a nurse specially trained to treat simple pathology or to give self-care advice. A specially trained GP supervises the nurses and GP assistants. Table 1 gives an overview of the number of professionals working at the IEPs and the control sites.

Control sites had to meet the following inclusion criteria: comparable number of patients visiting the A\&E department and GP post annually, comparable urbanization and proportion of immigrants in catchment area and comparable healthcare facilities in the direct environment. Major changes in the cooperation between the GP post and the hospital were considered an exclusion criterion. On the basis of these criteria, we selected two combinations of a GP post and A\&E department in Lelystad and Zaandam. Both cooperated with the study. Table 2 shows the characteristics of these sites.

\section{Outcome measures}

The outcome measures for patient flow were the total number of patients seen out of hours at the A\&E department, the GP post, or the IEP, time spent at the post, and percentage of self-referrals. Useful information about diagnosis was not available in the systems of the hospitals and this information was not reliable in the systems of the GP posts.

Table I: Numbers of staff working at the IEPs and control locations

\begin{tabular}{|c|c|c|c|c|c|c|c|c|}
\hline & \multicolumn{2}{|c|}{ IEP I } & \multicolumn{2}{|c|}{ Control I } & \multicolumn{2}{|c|}{ IEP 2} & \multicolumn{2}{|c|}{ Control 2} \\
\hline & 5-II PM & II PM-8 AM & $5-I I P M$ & II PM-8 AM & 5-II PM & II PM-8 AM & $5-I I P M$ & II PM-8 AM \\
\hline GP assistants & 3 & 2 & $2-4$ & 2 & $4-5$ & 2 & 3 & 2 \\
\hline GPs & 2 & I & $2-3$ & 1 & $2-3$ & I & $1-2$ & 1 \\
\hline A\&E doctors & 1 & 1 & 1 & 1 & $\mathrm{I}-2$ & 1 & I & 1 \\
\hline Nurses & $4-5$ & 3 & 3 & 2 & $3-4$ & 2 & $2-3$ & 2 \\
\hline
\end{tabular}

In some cases the number depends on the day of the week. 
Table 2: Characteristics of the settings (2006)

\begin{tabular}{|c|c|c|c|c|}
\hline & IEP I & Control I & IEP 2 & Control 2 \\
\hline Number of visits A\&ED yearly & $20-30.000$ & $20-30.000$ & $10-20.000$ & 10.20 .000 \\
\hline Number of contacts GP Post yearly & $20-30.000$ & $20-30.000$ & $10-20.000$ & 10.20 .000 \\
\hline Other hospital within $10 \mathrm{~km}$ & No & No & No & No \\
\hline Immigrants & $10-20 \%$ & $10-20 \%$ & $10-20 \%$ & $10-20 \%$ \\
\hline Urbanization (inhabitants per $\mathrm{km}^{2}$ ) & $>2000$ & $1000-2000$ & $<500$ & $<500$ \\
\hline
\end{tabular}

Sources: Central Office for Statistics, yearly reports hospitals and GP posts

The outcome measures for patient satisfaction were satisfaction about accessibility of the location, waiting time, reception, interpretation of the problem, treatment and information provided, autonomy, discharge and aftercare. The outcome measures for employee satisfaction were satisfaction regarding autonomy, clarity about their tasks, staffing, patient care, use of personal capacities, social climate, information, culture, work, and organization.

\section{Design and data collection}

Two IEPs (Purmerend and Haarlem) and two control sites (Lelystad and Zaandam) provided outcome information at $\mathrm{T}^{0}$, before the establishment of IEPs (Purmerend May 2005, Haarlem June 2006), and at $\mathrm{T}^{1}$, after the IEPs were established. The two IEPs recorded whether patients were seen by a GP/GP assistant or by an A\&E doctor/nurse. Table 3 gives an overview of the moments of data collection for the four sites.

\section{Patient flow}

At $\mathrm{T}^{0}$ retrospective data were retrieved from the information systems of the hospital and the GP post for patients seen Monday to Friday between $5 \mathrm{pm}$ and 8 am and on Saturday and Sunday in the first quarter of the year in which the IEP started and at $\mathrm{T}^{1}$ in the first quarter of the year after the IEP was established. The same quarters were chosen to avoid seasonal variation.

\section{Patient satisfaction}

Patient satisfaction was measured with a specially designed questionnaire based on a validated survey used in most Dutch hospitals [31] but adjusted for an IEP setting. A questionnaire was also designed for patients contacting the settings by phone. The survey for patients visiting the IEP, GP post or A\&E department, contained 45 questions that measured eight dimensions. The survey for patients contacting the IEP or GP post by phone, contained 27 questions that measured four dimensions. The patient satisfaction questionnaires were closed-ended questionnaires. Responses were given on a 5-point satisfaction scale ranging from "unsatisfied (1)" to "very satisfied (5)". The dimension scores were calculated as the mean item score. There was no retrospective information available about patient satisfaction.

The questionnaires were sent in May 2007 to the first 480 patients who had visited the IEP in a certain week and to the first 240 patients who had phoned the IEP. The same questionnaire was sent to the first 640 patients who had visited the control $A \& E$ departments and GP posts in the same week and to the first 160 patients who had phoned the control GP posts. After 10 days a reminder was sent. Questionnaires were returned by 151 patients who had visited the IEP (response 31\%), by 102 patients who had phoned the IEP (response 43\%), by 236 patients who had visited the control A\&E departments and GP posts (response 37\%), and by 57 patients who had phoned the GP Post (response 31\%).

\section{Employee satisfaction}

Employee satisfaction was measured with a specially developed survey based on a validated questionnaire frequently used in Dutch hospitals [32] but adjusted for an IEP setting. Employee satisfaction was measured on ten dimensions. In total, there were 61 questions. The dimension scores were calculated as the mean item scores. Responses were given on a 5-point satisfaction scale ranging from "I totally do not agree (1)" to "I totally agree (5)". There was no retrospective information available about employee satisfaction.

Table 3: Timetable for measurement of outcomes

\begin{tabular}{|c|c|c|c|c|c|c|c|c|}
\hline & \multicolumn{2}{|l|}{ IEP | } & \multicolumn{2}{|c|}{ Control I } & \multicolumn{2}{|l|}{ IEP 2} & \multicolumn{2}{|c|}{ Control 2} \\
\hline & $\mathbf{T}^{0}$ & $\mathbf{T}^{1}$ & $\mathbf{T}^{0}$ & $\mathbf{T}^{\prime}$ & $\mathbf{T}^{0}$ & $\mathbf{T}^{\prime}$ & $\mathbf{T}^{0}$ & $\mathbf{T}^{\prime}$ \\
\hline Patient flow & Q1 2005 & QI 2006 & QI 2005 & QI 2006 & Q1 2006 & QI 2007 & QI 2006 & QI 2007 \\
\hline Patient satisfaction & No & May 2007 & No & May 2007 & No & May 2007 & No & May 2007 \\
\hline Employee Satisfaction & No & May 2007 & No & May 2007 & No & May 2007 & No & May 2007 \\
\hline
\end{tabular}


All employees working at the IEP, A\&E department, or GP post were sent a questionnaire in May 2007. Because the number of A\&E doctors and GPs at the posts is flexible and not registered, the response rate is not known. In total, surveys were returned by 121 IEP employees and 103 employees working in the control settings. Further interviews were conducted with 44 randomly selected GPs, nurses, A\&E doctors, and GP assistants working in the different settings to get a more complete picture of the process of integration and the views and emotions of the staff.

As we only used routinely administrative registered data and questionnaires for this observational study, approval of an ethics committee was not necessary in the Netherlands.

\section{Results \\ Patient flow}

In total 6257 patients visited the A\&E department before the IEPs were established and 5715 were referred to an $A \& E$ doctor after the IEPs were established (Table 4). Over the same time period, the number of patients visiting the $\mathrm{A} \& \mathrm{E}$ departments in the control settings increased from 3985 to 4321 . In total 10195 patients visited a GP post before the IEPs were established and 12940 were seen by a GP, GP assistant or nurse after the IEPs were established. In the control settings, the number of patients visiting a GP post decreased from 14011 to 12719 .

Waiting/consultation times decreased from 1 hour and 56 minutes before the IEPs were established to 1 hour 42 minutes after the IEPs were established. In the control settings, waiting/consultation times increased from 1 hour and 34 minutes to 2 hours. The proportion of self-referrals decreased from $62 \%$ before the IEPs were established to $46 \%$ after they were established. In the control settings, the proportion of self-referrals increased from $53 \%$ to $58 \%$.

\section{Patient satisfaction}

Patients who visited the IEPs were not more satisfied than those who visited the control GP posts or A\&E departments (Table 5). However, patients who telephoned the IEP were more satisfied with the accessibility, interpreta- tion of the question, and information provided than were patients who telephoned the control GP posts (Table 6). In the Netherlands, it is not possible to telephone A\&E departments directly for triage and advice.

\section{Employee satisfaction}

Employees working at the IEPs were less satisfied than their colleagues working at GP posts or A\&E departments regarding their autonomy, the social climate, the information provided by the organization, the culture of the organization, their satisfaction with their work, and the possibility to use their own capacities and skills (see Table 7).

\section{Interviews}

Several conclusions could be drawn from the interviews. While almost all staff supported the idea of an IEP, they had problems with realizing the concept in practice. Several nurses and GP assistants were not satisfied about their increased responsibility; they did not choose this new profession, and several conditions such as a solid triage system were not fulfilled. Lastly, staff mentioned practical problems such as the GP post and the A\&E department still having separate meeting rooms, which did not facilitate integration.

\section{Discussion}

This study showed that the development of an IEP leads to a shift from secondary care to primary care, a decrease in waiting/consultation times, a decrease in self-referrals, and increased patient satisfaction with telephone contact with care providers. There was no difference in satisfaction between patients who visited an IEP and those who visited a GP post or A\&E department. In contrast, employees working at an IEP were less satisfied about their work and working conditions than employees working at GP posts or $\mathrm{A} \& \mathrm{E}$ departments.

However, it should be borne in mind that the integration of GP and hospital cultures and working style will take time, probably longer than the twelve months we allowed. Moreover, we collected data from only two locations, which limits the statistical power of this explorative research. The study had potential methodological limitations: the retrospective case-control design carried a risk of

Table 4: Patient flow in the IEP and control settings

\begin{tabular}{|c|c|c|c|c|}
\hline & \multicolumn{2}{|c|}{ IEP } & \multicolumn{2}{|c|}{ Control } \\
\hline & To & $T I$ & To & $T I$ \\
\hline Number of patients A\&E & 6257 & $5315 * *$ & 3985 & $4321^{*}$ \\
\hline Number of contacts GP Post & 10195 & $12940 *$ & $|40| \mid$ & $12719 * *$ \\
\hline Time spent at post & $\mathrm{I} \mathrm{hr} 56 \mathrm{~min}$ & $\mathrm{I} \mathrm{hr} 42 \mathrm{~min} * *$ & I hr 34 min & 2 hrs* \\
\hline$\%$ self-referrals & $62 \%$ & $46 \% * *$ & $53 \%$ & $58 \% *$ \\
\hline
\end{tabular}

$*$ TI significantly higher, $\mathrm{p}<0.05$, Chi square ** TI significantly lower, $\mathrm{p}<0.05$, Chi square 
Table 5: Patient satisfaction with IEPs compared with the control setting

\begin{tabular}{lcccc}
\hline & \multicolumn{2}{c}{ IEP } & \multicolumn{2}{c}{ Control } \\
& $n$ & mean & $n$ & mean \\
\hline Accessibility & 88 & 3.9 & 140 & 3.8 \\
Waiting time & 146 & 3.4 & 223 & 3.5 \\
Reception & 146 & 3.6 & 220 & 3.6 \\
Information and communication & 96 & 3.6 & 160 & 3.6 \\
Autonomy & 83 & 3.6 & 157 & 3.6 \\
Discharge and aftercare & 123 & 3.7 & 188 & 3.7 \\
Interpretation of the question & 135 & 3.7 & 216 & 3.8 \\
Treatment & 110 & 3.7 & 201 & 3.8 \\
\hline
\end{tabular}

( $I$ = not satisfied at all, 5 = very much satisfied $)$

confounding bias, selection bias, and information bias. These risks were minimized by careful selection of the control locations and protocols for uniform data collection. And finally, the response rates to the patient satisfaction surveys were lower than in most patient satisfaction surveys in the Netherlands (40-60\%). This restricts generalization for this part of our study.

The shift in patient flow was not caused by a change in patient population because patient characteristics (age, sex, and urgency of the health problem) were similar at $\mathrm{T}^{0}$ and $\mathrm{T}^{1}$. The decrease in the number of patients seen by $A \& E$ doctors at the IEP could have been caused by regional developments, such as negative publicity about the hospital and/or its A\&E department, changes in cooperation with ambulance services, or management policy decisions. However, no evidence for such problems was found during the interviews or during contact with the different sites.

Primary care costs less than hospital care, so the national introduction of IEPs could reduce national spending on health care. This aspect was not investigated in this study but it would be interesting to analyse the consequences of setting up IEPs on healthcare costs. On one hand, healthcare costs will be diminished by the shift from hospital care to primary care but on the other, the setting up of

Table 6: Patient satisfaction with telephone contact with the IEPs compared with the control GP Post

\begin{tabular}{lcccc}
\hline & \multicolumn{3}{c}{ IEP } & \multicolumn{2}{c}{ Control GP Posts } \\
& $n$ & mean & $n$ & mean \\
\hline Accessibility & 96 & $4.2^{*}$ & 53 & 3.9 \\
Interpretation of the question & 95 & $4.2^{*}$ & 52 & 3.9 \\
Information and communication & 91 & $3.7^{*}$ & 50 & 2.9 \\
Discharge and aftercare & 22 & 3.7 & 13 & 3.8 \\
\end{tabular}

* significantly higher $(\mathrm{p}<0.05)$, one-way ANOVA

( $I=$ not satisfied at all, $5=$ very much satisfied $)$
Table 7: Satisfaction of employees working in an IEP or control setting

\begin{tabular}{lcccc}
\hline & \multicolumn{2}{c}{ IEP } & \multicolumn{2}{c}{ Control } \\
& $n$ & mean & $n$ & mean \\
\hline Autonomy & 120 & 3.7 & 103 & $3.9^{*}$ \\
Clarity about task & 120 & 4.0 & 103 & 4.1 \\
Staffing & 120 & 3.1 & 103 & 3.0 \\
Patient care & 120 & 3.5 & 103 & 3.6 \\
Social climate & 120 & 3.5 & 103 & $3.8^{*}$ \\
Information & 120 & 3.2 & 103 & $3.4^{*}$ \\
Culture & 120 & 2.6 & 103 & $4.0^{*}$ \\
Work & 120 & 3.2 & 103 & $3.4^{*}$ \\
Organization & 120 & 4.0 & 103 & 4.2 \\
Use of personal capacities & 120 & 3.2 & 103 & $3.4^{*}$ \\
\hline
\end{tabular}

* significantly higher $(\mathrm{p}<0.05)$, one-way ANOVA

( $I$ = not satisfied at all, 5 = very much satisfied)

IEPs will require investment in staff training and numbers, and the provision of joint facilities, such as one check-in counter, one call-centre, and a meeting room. The latter may be necessary to bridge cultural differences between GP post employees and hospital employees.

The decrease in the number of self-referrals to A\&E departments after the IEPs were established was expected because a substantial proportion of self-referred patients with minor complaints were sent to the GP after triage and registered in the information system of the GP post.

The decrease in waiting/consultation times at the IEP compared with GP posts or A\&E departments is remarkable and could be due to improved triage and patient allocation in the IEPs, so that patients were seen by the most appropriate doctor. Other possible explanations for this decrease, such as changed patient characteristics and staff changes, are not likely since both were relatively stable during the study.

It is surprising that patients were not more satisfied with the IEP provision of care. Theoretically, setting up an IEP creates transparency; patients no longer have to decide whether to visit a GP or an A\&E doctor. Moreover, waiting/consultation times were shortened. This lack of satisfaction might be because most patients were seen by nurses rather than by doctors, and the single check-in desk may have comprised patient privacy. Another reason could be that most patients had not recently visited an emergency service and therefore could not compare their experiences with earlier ones in a traditional setting.

Employees at the IEPs were not satisfied about several aspects of their work. This is most likely due to the dramatic changes in work processes as a result of introducing new care professionals and a new triage protocol. Such 
changes can have considerable consequences. For example, nurses and GP assistants gained more autonomy and responsibility, but this was not always welcomed. Moreover, introduction of IEPs could have financial consequences for employees. In 2007, hospitals and their consultants were paid according to the number of patients seen at the A\&E department, yet with the development of IEPs A\&E doctors saw fewer patients, which could have had negative financial consequences. In one of the IEPs, the hospital and medical staff had an agreement with the health insurance company that their loss would be compensated. This was not the case in the other IEP.

The introduction of IEPs did not have consequences for the income of GPs or the GP posts.

Crucial to the success of IEPs is that patients are seen by an appropriate professional. This means that triage systems should be effective and preferably uniform. The two IEPs in this study used different triage systems, but a uniform triage system has recently been introduced for Dutch hospitals, GP services, ambulances, and mental health services and is currently being tested in four regions. If this so-called Dutch Uniform Triage System functions as expected, it will help IEPs achieve an efficient workflow and patient-centred care.

IEPs will not resolve all the problems of emergency services and may have some limitations. It will take many years to bridge the cultural gap between primary and secondary care professionals. Moreover, accessible, wellfunctioning IEPs may even create a demand for emergency services. In lowly populated regions, the provision of acute primary care by GPs might be more efficient than integrated care.

A prospective study with more IEPs and a longer followup is needed to determine whether IEPs are a welcome innovation that should be stimulated. That is why we are hoping to stimulate more hospitals and GP posts at home and abroad to establish IEPs. Only with more participants can we gather enough data to enable more definite conclusions to be drawn about this promising innovation.

\section{Conclusion}

IEPs could make emergency care more efficient. Patients are more satisfied with some aspects than they are with traditional GP posts and A\&E departments. However, professionals are not yet convinced about the advantages. Other GP posts and A\&E departments in different countries should be encouraged to set up IEPs.

\section{Competing interests}

The authors declare that they have no competing interests.

\section{Authors' contributions}

RBK designed the study, interpreted the results and drafted the manuscript. DJH and HCMK performed the analysis, interpreted the results and contributed to the discussion. All authors reviewed and edited the manuscript for intellectual content.

\section{Acknowledgements}

We thank the funding bodies of this research: the Central Office for Professions in Health Care (an independent advice body for the Dutch government), the health assurance company Achmea, and the Province of NorthHolland. We also thank the members of the advice committee, who met three times during the study. The authors appreciated the advice but bear the full responsibility for the content of this article. The authors are also totally independent from the funding parties. The advice committee including the funding bodies had no role at all in collecting, analysing and interpreting the data, in writing the manuscript, and in the decision to submit the article for publication.

Lastly, we thank Professor Jan Smit and Professor Jack Dekker, both from the Free University in Amsterdam, for giving methodological and textual advice during the preparation of this manuscript.

\section{References}

I. Schneider SM, Gallery ME, Schafermeyer R, Zwemer FL: Emergency department crowding: a point in time. Ann Emerg Med 2003, 42: $167-72$.

2. Roberts DC, McKay MP, Shaffer A: Increasing rates of emergency department visits for elderly patients in the United States, I 993 to 2003. Ann Emerg Med 2008, I:769-774.

3. Derlet R, Richards J, Kravitz R: Frequent overcrowding in US emergency departments. Acad Emerg Med 200I, 8: I5I-5.

4. Shah NM, Shah MA, Behbehani J: Predictors of non-urgent utilization of hospital emergency services in Kuwait. Soc Sci Med 1996, 42:1313-23.

5. Fatovich DM: Emergency medicine. BMJ 2002, 324:958-62.

6. Santos-Eggimann B: Increasing use of the emergency department in a Swiss hospital: observational study based on measures of the severity of cases. BMJ 2002, 324: I I86- I I87.

7. Moll van Charante EP, ter Riet G, Bindels P: Self-referrals to the A\&E department during out-of-hours: patients' motives and characteristics. Patient Educ Couns 2008, 70:256-65.

8. Hull S, Jones IR, Moser K, Fisher J: The use and overlap of AED and general practice services by patients registered at two inner London general practices. Br J Gen Pract I998, 48: I575-9.

9. Carret ML, Fassa AG, Kawachi I: Demand for emergency health service: factors associated with inappropriate use. $B M C$ Health Serv Res 2007, I 8: I 3 I.

10. Pereira S, Oliveira e Silva A, Quintas M, Almeida J, Marujo C, Pizarro $M$, et al.: Appropriateness of emergency department visits in a Portuguese university hospital. Ann Emerg Med 200I, 37:580-6.

II. Dale J, Green J, Reid F, Glucksman E: Primary care in the accident and emergency department: $I$. Prospective identification of patients. BM] I995, 3 I I:423-6.

12. Sempere-Selva T, Peiró S, Sendra-Pina P, Martínez-Espín C, LópezAguilera I: Inappropriate use of an accident and emergency department: magnitude, associated factors, and reasons-an approach with explicit criteria. Ann Emerg Med 200 I, 37:568-79.

13. David M, Schwartau I, Anand Pant H, Borde T: Emergency outpatient services in the city of Berlin: Factors for appropriate use and predictors for hospital admission. Eur J Emerg Med 2006, I 3:352-7.

14. Bianco A, Pileggi C, Angelillo IF: Non-urgent visits to a hospital emergency department in Italy. Public Health 2003, I I 7:250-5.

15. Lang T, Davido A, Diakité B, Agay E, Viel JF, Flicoteaux B: Nonurgent care in the hospital medical emergency department in France: how much and which health needs does it reflect? J Epidemiol Community Health 1996, 50:456-62. 
16. Afilalo M, Guttman A, Colacone A, Dankoff J, Tselios C, Beaudet M, et al: Emergency department use and misuse. J Emerg Med 1995, 13:259-64.

17. Petersen LA, Burstin HR, O'Neil AC, Orav EJ, Brennan TA: Nonurgent emergency department visits: the effect of having a regular doctor. Med Care 1998, 36:1249-55.

18. Lee A, Lau FL, Hazlett CB, Kam CW, Wong P, Wong TW, et al:: Factors associated with non-urgent utilization of accident and emergency services: a case-control study in Hong Kong. Soc Sci Med 2000, 5 I: 1075-85.

19. Giesen P, Franssen E, Mokkink H, Bosch W van den, van Vugt A, Grol $R$ : Patients either contacting a general practice cooperative or accident and emergency department out of hours: a comparison. Emerg Med J 2006, 23:73I-4.

20. Dale J, Green J, Reid F, Glucksman E, Higgs R: Primary care in the accident and emergency department: II. comparison of general practitioners and hospital doctors. BMJ 1995 3 I I:427-430.

21. Murphy AW, Plunkett PK, Bury G, Leonard C, Walsh J, Lynam F, et al.: Effect of patients seeing a general practitioner in accident and emergency on their subsequent reattendance: cohort study. BMJ 2000, 320:903-904.

22. Dale J, Lang H, Roberts JA, Green J: Glucksman: Cost effectiveness of treating primary care patients in accident and emergency: a comparison between general practitioners, senior house officers, and registrars. $B M]$ | 996, 3 | 2: | 340-4.

23. Murphy AW, Bury G, Plunkett PK, Gibney D, Smith M, Mullan E, et al: Randomised controlled trial of general practitioner versus usual medical care in an urban accident and emergency department: process, outcome, and comparative cost. $B M J$ | 996, 3 | 2: | |35-| |42

24. Ward P, Huddy J, Hargreaves S, Touquet R, Hurley J, Fothergill J: Primary care in London: an evaluation of general practitioners working in an inner city accident and emergency department. J Accid Emerg Med 1996, I3: I I-5.

25. Freeman GK, Meakin RP, Lawrenson RA, Leydon GM, Craig G: Primary care units in A\&E departments in North Thames in the 1990s: initial experience and future implications. Br J Gen Pract 1999, 49:107-10

26. Bury G, Hungerford $P$, Langton $D$, Plunkett $P$ : A \& E services in Ireland: the potential role of general practice in accident and emergency services. Ir J Med Sci 2000, 169:245-7.

27. van Uden CJ, Winkens RA, Wesseling G, Fiolet HF, van Schayck OC, Crebolder HF: The impact of a primary care physician cooperative on the caseload of an emergency department: the Maastricht integrated out-of-hours service. J Gen Intern Med 2005, 20:612-7.

28. van Uden C], Winkens RA, Wesseling G], Crebolder HF, van Schayck $C P$ : Use of out of hours services: a comparison between two organisations. Emerg Med J 2003, 20:184-7.

29. Roukema J, Steyerberg EW, van Meurs A, Ruige M, Lei J van der, Moll HA: Validity of the Manchester Triage System in paediatric emergency care. Emerg Med J 2006, 23:906-10.

30. Elshove-Bolk J, Mencl F, van Rijswijck BT, Simons MP, van Vugt AB Validation of the Emergency Severity Index (ESI) in selfreferred patients in a European emergency department. Emerg Med J 2007, 24: I70-4.

31. Engwirda-Kromdijk GJCM, Blijham GH, Veldkamp CMA, de Haes $\mathrm{H}$ [The development of a core questionnaire for patient satisfaction research in academic hospitals] Utrecht: Federation of Academic Hospitals; 2002.

32. van Horn JE: [Psychometric analysis of DOSB] Tilburg: Catholic University Brabant; 1993.

\section{Pre-publication history}

The pre-publication history for this paper can be accessed here:

http://www.biomedcentral.com/1472-6963/8/225/pre pub
Publish with Biomed Central and every scientist can read your work free of charge

"BioMed Central will be the most significant development for disseminating the results of biomedical research in our lifetime. "

Sir Paul Nurse, Cancer Research UK

Your research papers will be:

- available free of charge to the entire biomedical community

- peer reviewed and published immediately upon acceptance

- cited in PubMed and archived on PubMed Central

- yours - you keep the copyright
BioMedcentral 